\title{
Crossover from Diffusive to Ballistic Transport in Periodic Quantum Maps
}

\author{
Daniel K. Wójcik ${ }^{1,2}$ \\ Center for Nonlinear Science, School of Physics, Georgia Institute of Technology, \\ 837 State Street, Atlanta, GA 30332-0430, USA \\ Centrum Fizyki Teoretycznej Polskiej Akademii Nauk, Al. Lotników 32/46, 02-668 \\ Warszawa, Poland
}

J. R. Dorfman

Institute for Physical Science and Technology, and Department of Physics, University of Maryland, College Park, Maryland 20742

\begin{abstract}
We derive an expression for the mean square displacement of a particle whose motion is governed by a uniform, periodic, quantum multi-baker map. The expression is a function of both time, $t$, and Planck's constant, $\hbar$, and allows a study of both the long time, $t \rightarrow \infty$, and semi-classical, $\hbar \rightarrow 0$, limits taken in either order. We evaluate the expression using random matrix theory as well as numerically, and observe good agreement between both sets of results.

The long time limit shows that particle transport is generically ballistic, for any fixed value of Planck's constant. However, for fixed times, the semi-classical limit leads to diffusion. The mean square displacement for non-zero Planck's constant, and finite time, exhibits a crossover from diffusive to ballistic motion, with crossover time on the order of the inverse of Planck's constant.

We argue, that these results are generic for a large class of 1D quantum random walks, similar to the quantum multi-baker, and that a sufficient condition for diffusion in the semi-classical limit is classically chaotic dynamics in each cell. Some connections between our work and the other literature on quantum random walks are discussed. These walks are of some interest in the theory of quantum computation.
\end{abstract}

1 email: danek@cns.physics.gatech.edu

2 www: http://www.cns.gatech.edu/ danek/

Preprint submitted to Elsevier Science 30 October 2018 


\section{Introduction}

It is well known that the quantum properties of classically chaotic systems differ considerably from their classical versions $[1,2,3]$. The quantum dynamics of a classically chaotic system with a finite number of degrees of freedom is considerably more regular than its classical counterpart, as indicated by the structures developed by Wigner distributions in phase space for simple systems, and the fact that almost all definitions of the quantum mechanical version of the Kolmogorov-Sinai rate of entropy production give the value zero for finite quantum systems that are classically chaotic with positive KolmogorovSinai entropy [4]. Furthermore, it is often difficult to describe the behavior of quantum systems for both long times and for small values of Planck's constant, i.e. in the semi-classical regime. This difficulty is related to the fact that the long time limit and the limit of small Planck constant do not commute, in addition to analytical problems that often make explicit solutions of the equations for quantum systems difficult. Therefore it is of some interest to study simple models where both the quantum and classical properties are accessible to analytic and simple numerical studies, and where the two limits can be studied in detail. One example is the baker map. The classical version of this map is tractable analytically [5,6,7], and the quantum version is less so $[8,9]$, but easily studied using numerical methods.

It is even more challenging to study transport problems in detail. In recent years some attention has been devoted to connecting macroscopic transport properties with microscopic chaos, see e.g. [6,7] and other contributions to this volume. We find it of interest to study the quantum signatures of these relations and look for their appearance in the semi-classical regime. To gain intuition about the change in the character of transport properties from quantum to classical regime a convenient system to study is the uniform, periodic multi-baker map. Our interest in this map is stimulated by the fact that the classical version is a chaotic system with transport, in this case, diffusion, so it provides a model where one can study transport in the context of chaotic dynamics.

Both the classical $[10,11]$ and quantum [12,13] versions of the multi-baker map are based upon the baker map, which in its classical version is an area preserving, expanding and contracting transformation of the 2-torus onto itself. The multi-baker map is obtained by considering a two dimensional strip of unit height in the $y$-direction and a segment of the real $x$-axis, either of infinite length in both directions or of finite length with specified boundary conditions [10,11]. The multi-baker transformation is a combination of the baker map with a translation of points in each unit interval to corresponding points in the nearest intervals to the right or left according to a well defined prescription, to be given below. The quantum versions of these maps 
are obtained by means of a simple quantization where the $x$-axis is taken to correspond to position space, and the $y$-direction is taken to correspond to momentum space $[12,13]$. The quantum mechanics is obtained by requiring that there be an integral number of quantum states in a unit interval, and that the time development of these states correspond to the expanding and contracting properties of the classical map. Balazs and Voros [8], and Saraceno [9] were able to show that these requirements can be satisfied by means of a unitary operation on the quantum states, provided Planck's constant is taken to be the inverse of the number of quantum states in the unit square. Our work has been devoted to extending this quantum baker map to a quantum multi-baker map and examining the transport properties of this quantum map [12,13]. Other quantizations of multibaker maps as well as the Kapral-Elskens coupled baker model have been studied in [14]

In this paper we focus on the calculation of the mean square displacement (MSD) of a particle whose dynamics is described by a quantum multi-baker map. This quantity is of importance for the description of the average motion of a particle, since the time dependence of this quantity can distinguish between sub-diffusive, diffusive, and super-diffusive motion. A particular example of super-diffusive motion is, of course, ballistic motion characteristic of the motion of a free particle.

In the classical multi-baker, the MSD grows linearly in time, $\left\langle(\Delta r)^{2}\right\rangle=t$, characteristic of diffusion, for all times greater than some microscopic time. The quantum version shows an asymptotic ballistic, $t^{2}$, growth for what we will call uniform maps, which are translationally invariant. This is essentially the same as one finds in models of electron transport in periodic solids, such as the Krönig-Penney model [15]. One can find examples of non-translationally invariant multi-bakers where the particle is localized with no asymptotic growth of the MSD with time. Here we consider only translationally invariant models, and leave the large class of non-translationally invariant models for future work.

The classical multi-baker map $[10,11]$ is a simple model for deterministic diffusion along a one-dimensional lattice. It can easily be constructed to be isomorphic to a random walk with any given probabilities for jumping to the right and to the left along the lattice. The quantum multi-baker map $[12,13]$ is also an example of a random walk, but in this case, the walk is quantum mechanical and has very different properties from the classical map due to the interference of probability amplitudes for possible paths. The subject of quantum random walks has developed a literature over the past few years. Here we will also discuss the connection of the quantum multi-baker with other models of quantum walks. We mention now that most of the previous studies by other authors have been devoted to the so-called Hadamard walk [16], which is a special case of the quantum multi-baker for the largest allowed value of 
Planck's constant. Thus the multi-baker map studied here represents a generalization of the Hadamard walk to all allowed values of Planck's constant.

The plan of this paper is as follows. In Section 2 we present the basic equations defining the quantum multi-baker map and compare it to the classical version. In Section 3 we discuss the MSD for the quantum map, and in Section 4 show how random matrix theory may be used to evaluate the MSD for small values of Planck's constant, leaving some technical details for a longer paper [17]. In Section 5 we compare the results of random matrix theory with numerical studies of the map, which shows that random matrix theory is quite effective in reproducing the numerical results and in providing an expression showing the crossover from diffusive to ballistic motion for fixed, non-zero values of Planck's constant as the time becomes large. In Section 6 we discuss the connection between the multi-baker map and other models of quantum random walks. We conclude with a summary of results, and a discussion of the possible implications and extensions of this work.

\section{The quantum multi-baker map}

We begin with the classical multi-baker map $[5,10,11]$. It is a two-dimensional lattice system where the phase space at each lattice site is a square and the dynamics is a combination of transport of the phase space densities to neighboring cells, which models the free flight, followed by a local baker map evolution within a square, which models a collision with a fixed scatterer. That is, the multi-baker map, $M$, is a composition of two maps $M=B \circ T$ : transport, $T$, of phase points to neighboring cells, given by

$$
T(n, x, y)= \begin{cases}(n+1, x, y), & \text { for } 0 \leq x<1 / 2 \\ (n-1, x, y), & \text { for } 1 / 2 \leq x<1\end{cases}
$$

and the baker map, $B$, which acts on the $x, y$ coordinates of each cell, $n$, separately, according to

$$
B(n, x, y)= \begin{cases}(n, 2 x, y / 2), & \text { for } 0 \leq x<1 / 2 \\ (n, 2 x-1,(1+y) / 2), & \text { for } 1 / 2 \leq x<1\end{cases}
$$


The combination of these two maps is the multi-baker map which is a timereversible, measure preserving, chaotic transformation, with evolution law

$$
M(n, x, y)= \begin{cases}(n+1,2 x, y / 2), & \text { for } 0 \leq x<1 / 2 \\ (n-1,2 x-1,(1+y) / 2), & \text { for } 1 / 2 \leq x<1\end{cases}
$$

As mentioned above, this classical map represents a simple area-preserving model of simple random walk. It can also be considered a simplified Bernoulli map for the motion of light weakly interacting particles in a gas of heavy scatterers on regular lattice (periodic Lorentz gas).

The quantization of the multi-baker map is based upon the known quantization of the baker map on the unit square, carried out by Balazs and Voros, and by Saraceno $[8,9]$. We form the quantum multi-baker by transporting some of the quantum states to the next cell on the right and the others to the next cell on the left, based upon the way the transformed points are moved to neighboring cells in the classical multi-baker, and then model the scattering by the quantum baker map $B$ acting in every single cell.

To quantize the baker map, we regard the horizontal direction of the torus $[0,1)^{2}$ as the position axis, while vertical axis corresponds to the momentum direction. To obtain the Hilbert space we take the subspace of the wave functions on a line whose probability densities, $|\Psi(x)|^{2},|\tilde{\Psi}(p)|^{2}$ are periodic in both position and momentum representations, respectively: $\Psi(x+1)=\exp \left(i 2 \pi \varphi_{q}\right) \Psi(x)$, $\tilde{\Psi}(p+1)=\exp \left(i 2 \pi \varphi_{p}\right) \tilde{\Psi}(p)$, where $\varphi_{q}, \varphi_{p} \in[0,1)$ are phases parameterizing quantization. The quantization of the baker map requires the phase space volume to be an integer multiple of the quantum of action $[8,9,18,19,20,21]$. Therefore the effective Planck constant is $h=1 / N$, for a baker map on a unit torus, where $N$, an integer, is the dimension of the Hilbert space. The space and momentum representations are connected by a discrete Fourier transform $\left\langle p_{k} \mid q_{l}\right\rangle=\left[G_{N}\left(\varphi_{q}, \varphi_{p}\right)\right]_{k, l}:=N^{-1 / 2} \exp \left(-i 2 \pi N p_{k} q_{l}\right)$. The discrete positions and momenta are $q_{l}=\left(l+\varphi_{q}\right) / N, p_{k}=\left(k+\varphi_{p}\right) / N$, respectively. We take $N$ to be an even integer and label the quantum states in coordinate space by $j$, where $j=0,1, \ldots, N-1$. The states $j=0, \ldots N / 2-1$ are called the "left" states, with collective wave function, $\Psi_{L}$, while the remaining states, $j=N / 2, \ldots, N-1$ are called the "right" states, with wave function, $\Psi_{R}$. One may think of $\Psi_{R, L}$ as $N / 2$ component vectors.

Having constructed the Hilbert space, one looks for a family of unitary propagators parameterized by $N=1 / h$ which approach the classical map in semiclassical limit. The quantum baker map $[8,9,19]$ is then given by a combination of two operations. The first operation takes the $N / 2$ "left" states into momentum states labeled $k=0,1, \ldots, N / 2-1$, called "bottom" momentum states, by means of the $N / 2 \times N / 2$ Fourier transform matrix $G_{N / 2}\left(\varphi_{q}, \varphi_{p}\right)$. The "right" 
coordinate states are transformed to "top" momentum states in the same way. Now one has transformed $N$ spatial states into $N$ momentum states in a way that mimics the classical baker's map. The final step is to express the $N$ new states in coordinate representation by means of the $N \times N$ matrix, $G_{N}^{-1}$, which takes $N$ states in the momentum representation to their coordinate representation. The full baker transformation, $\mathbf{B}$, on the torus is then given by the unitary transformation

$$
\mathbf{B}:=G_{N}^{-1}\left[\begin{array}{cc}
G_{N / 2} & 0 \\
0 & G_{N / 2}
\end{array}\right]
$$

for even $N$. Other examples and discussions of issues concerning the quantization of area-preserving maps can be found for instance in $[8,9,18,19,20,21,22]$.

Using this transformation as a template we can easily express the quantum multi-baker map as a transformation of right and left quantum states in unit squares labeled by $n \pm 1$ to right and left states in the unit square labeled by $n, \Psi_{R, L}(n)$. That is, in the position representation the evolution of the wave function in the quantum multi-baker is given by the equations ${ }^{3}$

$$
\left[\begin{array}{c}
\Psi_{L}(n, t+1) \\
\Psi_{R}(n, t+1)
\end{array}\right]=G_{N}^{-1}(n) \cdot\left[\begin{array}{cc}
G_{N / 2}(n) & 0 \\
0 & G_{N / 2}(n)
\end{array}\right] \cdot\left[\begin{array}{c}
\Psi_{L}(n-1, t) \\
\Psi_{R}(n+1, t)
\end{array}\right]
$$

where $G_{N}(n)$ is the discrete Fourier transform

$$
\left(G_{N}(n)\right)_{k j}=\frac{1}{\sqrt{N}} e^{-2 \pi i\left(k+\varphi_{p}(n)\right)\left(j+\varphi_{q}(n)\right) / N}
$$

In principle, the Fourier transformation matrix $G_{N}(n)$ can depend upon the cell index $n$. Here we will consider the case where this matrix is independent of the cell index, so that the system of equations is translationally invariant from cell to cell. We restrict our attention here to finite systems of length $L$ with periodic boundary conditions. This condition induces Bloch states and an eventual ballistic motion of particles through the chain. We associate $L$ dimensional Hilbert space with the lattice, and since the internal space is $\mathbb{C}^{N}$, the Hilbert space of the system is the tensor product $\mathbb{C}^{L} \otimes \mathbb{C}^{N}$. We will

3 Note that the construction we use here differs slightly from the one we studied in our previous work [12]. We change it to make the construction consistent with the general picture that we now have as well as to make model clearer. The properties of the two versions of random model should be the same, and the periodic model is unaffected. 
work with the basis defined by $|n, \pm, i\rangle$, where $n$ is the lattice site, \pm denotes left/right half of the square, and $i=0, \ldots, N / 2-1$ denotes the state in given half in the position representation. Since the states associated with the left half of the cell go one step to the right we write $\Psi_{L} \equiv \Psi_{+}$, accordingly $\Psi_{R} \equiv \Psi_{-}$. Then the general wave function for the multi-baker chain can be written as

$$
|\Psi\rangle=\sum_{n} \sum_{\varepsilon= \pm}\left|\Psi_{\varepsilon}(n)\right\rangle
$$

where $\left|\Psi_{\varepsilon}(n)\right\rangle:=P_{\varepsilon}(n)|\Psi\rangle, P_{\varepsilon}(n):=\sum_{i}|n, \varepsilon, i\rangle\langle n, \varepsilon, i|$ is orthogonal projection of the state vector onto the "left" or "right" subspace at site $n$. Thus the inner product takes form

$$
\langle\Phi \mid \Psi\rangle:=\sum_{n} \sum_{\varepsilon= \pm}\left\langle\Phi_{\varepsilon}(n) \mid \Psi_{\varepsilon}(n)\right\rangle
$$

We may think of $\left|\Psi_{\varepsilon}(n)\right\rangle$ and $\left\langle\Psi_{\varepsilon}(n)\right|$ as corresponding to $N / 2$-component column and row vectors, respectively, while $|\Psi\rangle$ corresponds to an $N L$-component vector. Since we work mostly in position basis, we use $N / 2$ dimensional vectors $\Psi_{\varepsilon}(n)$, whose $i$-th components are $\Psi_{ \pm}(n, i)=\langle n, \pm, i \mid \Psi\rangle$, where the index $n$ denotes the lattice site of the cell under consideration, as above, the notation \pm denotes the particular half of the unit square under consideration, the left half indicated by + moves to the cell to the right, and the right half, denoted by - , moves to the cell to the left. The index $i$ denotes a particular quantum state in the right or left half of the unit cell, specified by \pm and $i=0,1, \ldots, N / 2-1$. The action of the quantum multi-baker map on the total wave function will be written as the action of an operator $\mathbf{M}$ defined by Eq. $(2)$, as

$$
\left|\Psi^{\prime}\right\rangle=\mathbf{M}|\Psi\rangle
$$

The operator $\mathbf{M}$ is the Floquet operator for the multi-baker map and will be used to determine the time dependence of various observables for this system, as

$$
\Omega(t)=\mathbf{M}^{\dagger t} \Omega \mathbf{M}^{t}
$$

where $\Omega$ is any observable of the system.

The complete specification of the model requires values for the phases $\varphi_{q}, \varphi_{p}$. There is a considerable amount of freedom for choosing these phases. As mentioned above, one may choose them to vary from cell to cell. A random variation of phases from cell to cell produces a disordered multi-baker map, while requiring that the phases have constant values throughout the lattice produces 
a regular, or uniform multi-baker map which is translationally invariant from one cell to the next. Many other choices are possible and can be of interest. Here we consider only the translationally invariant case. In numerical calculations we use the values for the phases chosen by Balazs and Voros [8] with $\varphi_{q}=\varphi_{p}=0$; those used by Saraceno [9] $\varphi_{q}=\varphi_{p}=1 / 2$, which lead to survival of additional classical symmetry in the model, as well as more generic values. We will see below that the Saraceno phases lead to a non-generic behavior for the MSD.

Let us consider now the structure of eigenstates of the unitary operator $\mathbf{M}$. Since we consider periodic boundary conditions every eigenstate corresponding to the eigenvalue $\lambda=e^{i \kappa}$ has a Bloch form

$$
\Psi(n, \pm)=\exp (i \kappa n) \tilde{\Psi}_{ \pm} / \sqrt{L}
$$

where $\left[\begin{array}{c}\tilde{\Psi}_{+} \\ \tilde{\Psi}_{-}\end{array}\right]$is the normalized eigenstate of a modified quantum baker operator

$$
G_{N}^{-1}\left[\begin{array}{cc}
G_{N / 2} e^{-i \kappa} & 0 \\
0 & G_{N / 2} e^{i \kappa}
\end{array}\right]
$$

Periodic boundary conditions imply $e^{i \kappa L}=1$, thus we have $\kappa_{k}=2 \pi k / L, k=$ $0,1, \ldots, L-1$. For every $\kappa_{k}$ we have $N$ eigenstates. We will enumerate the eigenstates of $M$ by $k, n$, with $n=0, \ldots, N-1$. Thus $\Psi_{n}^{k}$ is the eigenstate given by the ansatz (8) with $\kappa_{k}=2 \pi k / L$, corresponding to the eigenvalue $e^{i \varphi_{n}^{k}}$, where phases $\varphi_{n}^{k}$ are counted for a given $k$ from 0 (including) to $2 \pi$, i.e. $0 \leq \varphi_{n}^{k} \leq \varphi_{n+1}^{k}<2 \pi$.

\section{The mean square displacement (MSD) in the uniform multi- baker map}

In order to formulate our calculation of the MSD for the uniform multi-baker map in the most convenient way, we introduce two operators, $r, v$, which represent a coarse position operator and a coarse velocity operator, respectively. These operators are coarse in the sense that $r$ simply gives the lattice site associated with a particular quantum state, and the coarse grained velocity $v$ is given by $v=\mathbf{M}^{\dagger} r \mathbf{M}-r$. We argue elsewhere [17], that for a translationally invariant system $v$ has a very simple form with values \pm 1 , given by the change in cell index for each quantum state. Thus 


$$
\begin{aligned}
& r|n, \pm, i\rangle=n|n, \pm, i\rangle \\
& v|n, \pm, i\rangle= \pm|n, \pm, i\rangle
\end{aligned}
$$

so that explicit expressions for the operators $r, v$ are

$$
\begin{aligned}
& r=\sum_{n, \varepsilon, i} n|n, \varepsilon, i\rangle\langle n, i, \varepsilon|, \\
& v=\sum_{n, \varepsilon, i} \varepsilon|n, \varepsilon, i\rangle\langle n, \varepsilon, i| .
\end{aligned}
$$

Suppose we prepare the system in a pure state $|\Psi\rangle$. Then the mean square displacement of the particle starting in this state is given by

$$
\left\langle(\Delta r)^{2}(t)\right\rangle_{\Psi}=\left\langle\left(\mathbf{M}^{\dagger t} r \mathbf{M}^{t}-r\right)^{2}\right\rangle_{\Psi}=\left\langle\left(\sum_{\tau=0}^{t-1} v_{\tau}\right)^{2}\right\rangle_{\Psi}=\sum_{\tau_{1}, \tau_{2}=0}^{t-1}\left\langle v_{\tau_{1}} v_{\tau_{2}}\right\rangle_{\Psi}
$$

where $\langle A\rangle_{\Psi}:=\langle\Psi|A| \Psi\rangle=\operatorname{Tr}(|\Psi\rangle\langle\Psi| A)$. Depending on the original state we have a distribution of possible results. To characterize it we can calculate its average over all the possible initial states, and the root mean square deviation from the average, which quantifies the spread of the results, or the quality of the prediction based on the average. In this Section we find the expressions for both the equilibrium MSD as well as for the equilibrium fluctuations of this function. In the next section we approximate the average results using random matrix theory, and then we compare them with numerical evaluation of the exact formulas.

Since the average over all the possible pure states gives the most incoherent mixture, we obtain a simple expression for the equilibrium MSD as

$$
\left\langle(\Delta r)^{2}(t)\right\rangle=\sum_{\tau_{1}, \tau_{2}=0}^{t-1}\left\langle v_{\tau_{1}} v_{\tau_{2}}\right\rangle
$$

where

$$
\langle A\rangle:=\operatorname{Tr}\left(\varrho_{\mathrm{eq}} A\right)=\frac{1}{L N} \operatorname{Tr}(A),
$$

$L$ is the length of the chain (assume periodic boundary conditions), and $N$ is the dimension of the Hilbert space for a single cell. Clearly, $\varrho_{\text {eq }}=1_{N L} /(L N)$ is the equilibrium state for all the QMB, that is, it is time invariant

$$
\mathbf{M} \varrho_{\mathrm{eq}} \mathbf{M}^{\dagger}=\varrho_{\mathrm{eq}}
$$


and it maximizes the entropy $S=-\operatorname{Tr}(\varrho \ln \varrho)$. Time invariance implies that the velocity autocorrelation function

$$
C_{\tau_{1}, \tau_{2}}:=\left\langle v_{\tau_{1}} v_{\tau_{2}}\right\rangle=\left\langle\mathbf{M}^{\dagger \tau_{1}} v \mathbf{M}^{\tau_{1}} \mathbf{M}^{\dagger \tau_{2}} v \mathbf{M}^{\tau_{2}}\right\rangle
$$

satisfies $C_{\tau_{1}, \tau_{2}}=C_{\tau_{1}-\tau_{2}, 0}=C_{0, \tau_{1}-\tau_{2}} \equiv C_{\tau_{1}-\tau_{2}}$. Thus we can write

$$
\left\langle(\Delta r)^{2}(t)\right\rangle=\sum_{\tau=0}^{t-1} C_{0}+2 \sum_{\tau_{1}>\tau_{2}=0}^{t-1} C_{\tau_{1}-\tau_{2}}=C_{0} t+2 \sum_{\tau=1}^{t-1}(t-\tau) C_{\tau} .
$$

This leads to a Green-Kubo like formula for the diffusion coefficient

$$
D=\lim _{t \rightarrow \infty} \frac{1}{2 t}\left\langle(\Delta r)^{2}(t)\right\rangle=\frac{1}{2} C_{0}+\sum_{\tau=1}^{\infty} C_{\tau}=\sum_{\tau=0}^{\infty} C_{\tau}-\frac{1}{2} C_{0},
$$

paralleling the classical case. However, here the diffusion coefficient turns out to be infinite.

Thus the MSD is written as the sum of time correlations of the velocity, just as in the classical case, but the difference in mechanics will lead to important differences in the time development of the MSD.

The translational invariance of the system, together with the uniform equilibrium density, imply that instead of summing over all the $N$ quantum states in the $L$ cells, we can sum only over the $N$ quantum states in one cell, see Ref. [17]. Thus we can write

$$
\begin{aligned}
C_{\tau} & =\frac{1}{L N} \operatorname{Tr}\left[\mathbf{M}^{\dagger \tau} v \mathbf{M}^{\tau} v\right] \\
& =\frac{1}{N} \sum_{i, \varepsilon= \pm}\left\langle n=0, \varepsilon, i\left|\mathbf{M}^{\dagger \tau} v \mathbf{M}^{\tau} v\right| n=0, \varepsilon, i\right\rangle \\
& =\frac{1}{N} \sum_{i, \varepsilon= \pm} \varepsilon\left\langle n=0, \varepsilon, i\left|\mathbf{M}^{\dagger \tau} v \mathbf{M}^{\tau}\right| n=0, \varepsilon, i\right\rangle .
\end{aligned}
$$

Thus we have to sum, with appropriate signs, over all the possible cyclic paths starting in a fixed cell. The next simplification provided by the translational invariance of the model is that we can replace the unitary operator $\mathbf{M}$ for the full multi-baker in Eq. (18) by the operator B acting within a unit cell. This replacement follows from the observation that the velocity operator is diagonal in the representation based on the states in the cells, with a block structure that is identical from one cell to the next ${ }^{4}$. Therefore, the velocity operator at

$\overline{4}$ This would not be the case for the disordered multi-baker maps where the quantization phases vary from one cell to the next. 
time step $t$ takes on the same value in all of quantum states that are periodic images of any state in a fixed cell. Thus we can express the velocity correlation function, $C_{\tau}$, as

$$
C_{\tau}=\frac{1}{N} \operatorname{Tr}\left[\mathbf{B}^{\dagger \tau} \mathbf{J B}^{\tau} \mathbf{J}\right]
$$

where the matrix $\mathbf{J}$ is the velocity operator restricted to a single cell, in position representation given by

$$
\mathbf{J}=\left[\begin{array}{cc}
1_{N / 2} & 0 \\
0 & -1_{N / 2}
\end{array}\right]
$$

Here $1_{N / 2}$ is the $N / 2 \times N / 2$ unit operator.

To proceed further we must now use specific properties of the quantum baker map B, and in particular, its spectral properties. Since $\mathbf{B}$ is a unitary operator, its eigenvalues lie on the unit circle and define a set of $N$ phases, $\varphi_{j}$ or quasienergies. We will denote the corresponding eigenstates by the Dirac kets $|j\rangle$. Thus the spectral problem for the operator $\mathbf{B}$ is

$$
\mathbf{B}|j\rangle=e^{i \varphi_{j}}|j\rangle
$$

Then we can express the velocity correlation function $C_{\tau}$ as

$$
\begin{aligned}
C_{\tau} & =\frac{1}{N} \sum_{j, k} e^{-i \varphi_{k} \tau}\langle k|\mathbf{J}| j\rangle e^{i \varphi_{j} \tau}\langle j|\mathbf{J}| k\rangle \\
& =\frac{1}{N} \sum_{j, k}\left|\mathbf{J}_{j k}\right|^{2} e^{i\left(\varphi_{j}-\varphi_{k}\right) \tau} \\
& =\frac{1}{N}\left[\sum_{j}\left|\mathbf{J}_{j j}\right|^{2}+2 \sum_{j>k}\left|\mathbf{J}_{j k}\right|^{2} \cos \left(\varphi_{j}-\varphi_{k}\right) \tau\right] .
\end{aligned}
$$

The matrix elements of $\mathbf{J}$ satisfy

$$
\sum_{j, k}\left|\mathbf{J}_{j k}\right|^{2}=\operatorname{Tr} \mathbf{J}^{2}=N=\sum_{j}\left|\mathbf{J}_{j j}\right|^{2}+\sum_{j>k} 2\left|\mathbf{J}_{j k}\right|^{2} .
$$

Therefore

$$
C_{\tau}=\frac{1}{N}\left[N+\sum_{j>k}\left|\mathbf{J}_{j k}\right|^{2}\left(e^{i\left(\varphi_{j}-\varphi_{k}\right) \tau}+e^{-i\left(\varphi_{j}-\varphi_{k}\right) \tau}-2\right)\right]
$$




$$
=1-\frac{4}{N} \sum_{j>k}\left|\mathbf{J}_{j k}\right|^{2} \sin ^{2} \frac{\left(\varphi_{j}-\varphi_{k}\right) \tau}{2}
$$

We can now substitute these results into Eq. (17) for the mean square displacement to obtain

$$
\begin{aligned}
\left\langle(\Delta r)^{2}(t)\right\rangle & =t+\sum_{\tau=1}^{t-1}(t-\tau) C_{\tau} \\
& =t+t(t-1) \frac{1}{N} \sum_{j}\left|\mathbf{J}_{j j}\right|^{2}+\frac{4}{N} \sum_{j>k}\left|\mathbf{J}_{j k}\right|^{2} \sum_{\tau=1}^{t-1} \tau \Re e^{i\left(\varphi_{j}-\varphi_{k}\right)(t-\tau)}(25
\end{aligned}
$$

The last expression is particularly useful for averaging and will be used in the next section.

The sums over the intermediate times $\tau$ in Eq. (25) can easily be carried out. We assume now that there is no degeneracy in the spectrum of quasi-energies and, after some algebra, we obtain an expression for the MSD as

$$
\begin{aligned}
\left\langle(\Delta r)^{2}(t)\right\rangle & =t^{2} \frac{1}{N} \sum_{j}\left|\mathbf{J}_{j j}\right|^{2}+\frac{2}{N} \sum_{j>k}\left|\mathbf{J}_{j k}\right|^{2} \frac{\sin ^{2} \frac{\left(\varphi_{j}-\varphi_{k}\right) t}{2}}{\sin ^{2} \frac{\varphi_{j}-\varphi_{k}}{2}} \\
& =\frac{1}{N} \sum_{j, k}\left|\mathbf{J}_{j k}\right|^{2} \frac{\sin ^{2} \frac{\left(\varphi_{j}-\varphi_{k}\right) t}{2}}{\sin ^{2} \frac{\varphi_{j}-\varphi_{k}}{2}}
\end{aligned}
$$

Whenever two eigenphases $\varphi_{j}, \varphi_{k}$ are equal, the contribution to the sum $\frac{\sin ^{2} \frac{\left(\varphi_{j}-\varphi_{k}\right) t}{2}}{\sin ^{2} \frac{\varphi_{j}-\varphi_{k}}{2}}$ must be replaced by $t^{2}$.

We see that there is typically a ballistic contribution coming from diagonal and possibly some degenerate terms. The other contributions are oscillatory and usually negligible in the long time limit. It can happen, however, that the ballistic contribution disappears altogether and we have only oscillations which means that the particle is localized. An example is provided in Section 5 and an explanation in more general context is given in the final Section. It is interesting, that the obtained result is completely independent of the length of the system. This is not true for the fluctuations of the MSD.

It is not easy to evaluate this expression, Eq. (27), analytically. Therefore in section 4 we present an approximation based on the random matrix theory, and in Section 5 we evaluate it numerically for some particular choices of parameters, and compare it with the RMT averages. 


\subsection{Fluctuations in the MSD}

We have computed the MSD as an equilibrium average where all eigenstates have the same weight. One might ask about the dependence of the average square displacement for an individual quantum state. In order to say something about the fluctuations of the average square displacement from one state to the next, we now consider, $\Delta_{\Psi} r^{2}(t)$ the average square displacement for the quantum state $|\Psi\rangle$, where $\Delta_{\Psi} r^{2}(t)=\left\langle\Psi\left|\Delta r^{2}(t)\right| \Psi\right\rangle$. We will try to characterize the fluctuations of the square displacement among various quantum states by calculating, in so far as it is possible, the mean square fluctuation of $\Delta_{\Psi} r^{2}(t)$, which we denote by $\Delta^{2}(t)$, where

$$
\Delta^{2}(t)=\left\langle\left[\Delta_{\Psi} r^{2}(t)-\left\langle\Delta r^{2}(t)\right\rangle\right]^{2}\right\rangle_{\Psi}
$$

The average $\langle A(\Psi)\rangle_{\Psi}$ is defined as follows: if $\left|\Psi_{\alpha}\right\rangle$ is an arbitrary orthonormal basis and $b_{\alpha}:=\left\langle\Psi_{\alpha} \mid \Psi\right\rangle$ are the complex coefficients of expansion of $|\Psi\rangle$ in this basis then

$$
\langle A(\Psi)\rangle_{\Psi}:=\frac{\int d^{2} b_{1} \ldots d^{2} b_{L N} \delta\left(1-\sum_{\alpha=1}^{N L}\left|b_{\alpha}\right|^{2}\right) A(\Psi)}{\int d^{2} b_{1} \ldots d^{2} b_{L N} \delta\left(1-\sum_{\alpha=1}^{N L}\left|b_{\alpha}\right|^{2}\right)}=\frac{\int d \Omega_{2 L N} A(\Psi)}{\int d \Omega_{2 L N}}
$$

Thus it is an average over $2 L N$-dimensional sphere.

Before we calculate the right hand side of Eq. (28), we note that the equilibrium mean square displacement can be written in a very similar way as an average over the space of all the states

$$
\left\langle(\Delta r(t))^{2}(t)\right\rangle=\left\langle\Delta_{\Psi} r^{2}(t)\right\rangle_{\Psi}
$$

Indeed, using Eq. (29) we obtain

$$
\left\langle(\Delta r(t))^{2}(t)\right\rangle=\sum_{\alpha, \beta}\left\langle b_{\alpha}^{*} b_{\beta}\right\rangle\left\langle\psi_{\alpha}\left|\Delta r^{2}(t)\right| \psi_{\beta}\right\rangle
$$

Only contributions from $\alpha=\beta$ survive due to phase averaging, and the diagonal terms carry the same contribution, therefore $\left\langle b_{\alpha}^{*} b_{\beta}\right\rangle_{\Psi}=\delta_{\alpha, \beta} /(N L)$. If we insert this relation into the right hand side of Eq. (31), we recover Eq. (14) with the quantity $A=\Delta r^{2}(t)$.

Returning to Eq. (28), we see that the right hand side can be written as 


$$
\begin{aligned}
\Delta^{2}(t)= & \sum_{n, m, k, l=0}^{t-1} \sum_{\alpha, \beta, \gamma, \delta=1}^{L N}\left\langle b_{\alpha}^{*} b_{\beta} b_{\gamma}^{*} b_{\delta}\right\rangle_{\Psi}\left\langle\Psi_{\alpha}\left|v_{n} v_{m}\right| \Psi_{\beta}\right\rangle\left\langle\Psi_{\gamma}\left|v_{k} v_{l}\right| \Psi_{\delta}\right\rangle \\
& -\left[\left\langle\Delta r^{2}(t)\right\rangle\right]^{2} .
\end{aligned}
$$

Observe that if at least one phase, or equivalently one subscript, is unpaired, the average $\left\langle b_{\alpha}^{*} b_{\beta} b_{\gamma}^{*} b_{\delta}\right\rangle$ will be zero. Therefore the average of the four coefficients is non-zero only if the indices are paired. This can be arranged in three ways, so that

$$
\left\langle b_{\alpha}^{*} b_{\beta} b_{\gamma}^{*} b_{\delta}\right\rangle=c_{1} \delta_{\alpha \beta} \delta_{\gamma \delta}\left(1-\delta_{\beta \gamma}\right)+c_{1} \delta_{\alpha \delta} \delta_{\gamma \beta}\left(1-\delta_{\alpha \beta}\right)+c_{2} \delta_{\alpha \beta} \delta_{\gamma \delta} \delta_{\alpha \gamma}
$$

where

$$
\begin{aligned}
& c_{1}=\left\langle\left|b_{1}\right|^{2}\left|b_{2}\right|^{2}\right\rangle \\
& c_{2}=\left\langle\left|b_{1}\right|^{4}\right\rangle
\end{aligned}
$$

Let $A=\sum_{m, n=0}^{t-1} v_{m} v_{n}$. Then

$$
\begin{aligned}
\Delta^{2}(t)= & c_{1} \sum_{\alpha \neq \beta}\left\langle\Psi_{\alpha}|A| \Psi_{\alpha}\right\rangle\left\langle\Psi_{\beta}|A| \Psi_{\beta}\right\rangle+c_{1} \sum_{\alpha \neq \beta}\left\langle\Psi_{\alpha}|A| \Psi_{\beta}\right\rangle\left\langle\Psi_{\beta}|A| \Psi_{\alpha}\right\rangle \\
& +\sum_{\alpha} c_{2}\left\langle\Psi_{\alpha}|A| \Psi_{\alpha}\right\rangle\left\langle\Psi_{\alpha}|A| \Psi_{\alpha}\right\rangle-\left[\frac{1}{N L} \operatorname{Tr} A\right]^{2} \\
= & c_{1}(\operatorname{Tr} A)^{2}+c_{1} \operatorname{Tr} A^{2}+\sum_{\alpha}\left(c_{2}-2 c_{1}\right)\left|\left\langle\Psi_{\alpha}|A| \Psi_{\alpha}\right\rangle\right|^{2}-\left[\frac{1}{N L} \operatorname{Tr} A\right]^{2}
\end{aligned}
$$

Since the term $\sum_{\alpha}\left|\left\langle\Psi_{\alpha}|A| \Psi_{\alpha}\right\rangle\right|^{2}$ is basis dependent we must have $c_{2}=2 c_{1}$. Also,

$$
\sum_{\alpha, \beta}\left|b_{\alpha}\right|^{2}\left|b_{\beta}\right|^{2}=\left\langle\sum_{\alpha, \beta}\left|b_{\alpha}\right|^{2}\left|b_{\beta}\right|^{2}\right\rangle=1
$$

Since there are $L N$ quantum states, this reduces to

$$
c_{2} L N+c_{1} L N(L N-1)=1
$$

therefore

$$
c_{1}=\frac{1}{L N(L N+1)} \quad c_{2}=\frac{2}{L N(L N+1)} .
$$

To proceed further we need to use the results obtained earlier following Eq. (9) for the eigenstates and eigenphases of the multi-baker operator. The eigenstates have the form of periodic Bloch states and the eigenphases appear in $L$ 
sets of $N$ eigenphases. After some lengthy, but straightforward, calculations one finds the final result for the fluctuations of the average square deviations as $[17]$

$$
\begin{aligned}
\Delta^{2}(t)= & c_{1}\left[(\operatorname{Tr} A)^{2}+\operatorname{Tr} A^{2}\right]-\frac{1}{L^{2} N^{2}}(\operatorname{Tr} A)^{2} \\
= & c_{1}\left[\operatorname{Tr} A^{2}-\frac{1}{L N}(\operatorname{Tr} A)^{2}\right] \\
= & \frac{1}{L+1 / N} \frac{1}{N^{2}}\left[\left\langle\sum_{p, s=0}^{N-1}\left|\sum_{r=0}^{N-1} J_{p r}^{a} J_{r s}^{a} \frac{\sin \frac{\left(\varphi_{p}^{a}-\varphi_{r}^{a}\right) t}{2}}{\sin \frac{\varphi_{p}^{a}-\varphi_{r}^{a}}{2}} \frac{\sin \frac{\left(\varphi_{r}^{a}-\varphi_{s}^{a}\right) t}{2}}{\sin \frac{\varphi_{r}^{a}-\varphi_{s}^{a}}{2}}\right|^{2}\right\rangle_{a}\right. \\
& \left.-\frac{1}{N}\left(\sum_{m, n=0}^{N-1}\left|J_{n m}\right|^{2} \frac{\sin ^{2} \frac{\left(\varphi_{n}-\varphi_{m}\right) t}{2}}{\sin ^{2} \frac{\varphi_{n}-\varphi_{m}}{2}}\right)^{2}\right]
\end{aligned}
$$

The main source for the system size, $L$, dependence of this fluctuation result is the factor of $L^{-1}$ in front of the whole expression. This provides the usual $L^{-1 / 2}$ decay of the root mean square fluctuation which suggests that in the large systems the equilibrium average is typical for almost every initial, pure state. The eigenphases $\varphi_{j}^{a}$ also depend on the system size, so that in the absence of a further evaluation of the sums, one cannot precisely determine the size dependence of $\Delta^{2}(t)$, however, it is reasonable to expect that the contributions for different Bloch vectors are of the same order. Numerical results suggest that this expectation is correct for the quantum multi-bakers, and the final result for the right hand side of Eq. (40) decays inversely with $L$. This is shown in Figure 1. The left picture shows the time and size dependence of the relative fluctuations $\frac{\sqrt{\Delta^{2}(t)}}{\left\langle\Delta r^{2}(t)\right\rangle}$ on a log-log plot. The right picture shows the same data with each curve multiplied by square root of $L$. The scaling is remarkable. Plots were obtained through numerical evaluation of exact formula (40) for $N=50, \varphi_{q}=\varphi_{p}=0$. Using the methods of random matrix theory discussed
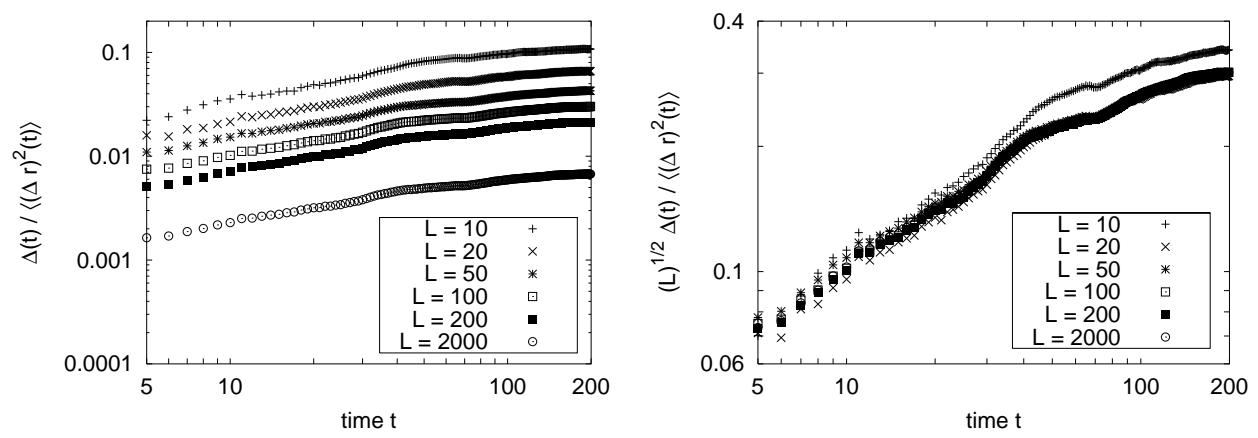

Fig. 1. Time and length dependence of the relative fluctuations $\frac{\sqrt{\Delta^{2}(t)}}{\left\langle\Delta r^{2}(t)\right\rangle}$. The left picture shows log-log plot of the unscaled data, the right picture shows each curve multiplied by square root of $L$. Plots made for $N=50, \varphi_{q}=\varphi_{p}=0$.

in the next section, in the limit of large $N$, one can replace the average over 
the Bloch states, labeled by the subscript $a$, by averages over random matrix ensembles. We have not yet done this and leave it for future work.

\section{Random matrix theory for the mean square displacement}

To proceed further we need to know the quasi-energies and eigenvectors of the operator B. For large $N$ it is not possible to determine these quantities by analytical means. Instead one uses numerical methods to diagonalize $\mathbf{B}$. The results obtained this way will be discussed in the next section. Here we will show how random matrix theory $[2,23]$ may be used to evaluate the approximate value of the MSD. In a sense, the use of random matrix theory must be considered to be a kind of uncontrolled approximation method since there are no theorems indicating that the distributions of quasi-energies are described by any of the three random matrix ensembles, and there are no similar results for the eigenvectors. However, since the classical baker map is an example of a strongly chaotic system, we can expect that one of the two ensembles, the circular orthogonal ensemble, COE, or the circular unitary ensemble, CUE, might provide a very good approximation when it is used to evaluate the right hand sides of Eq. (27), see ref. [9,24]. The results obtained this way will, of course, be averages over the ensemble of random matrices, and not characteristic of any one matrix in the ensemble, and perhaps not of the baker map itself. We will see shortly that the numerical results for the baker map are in very good agreement with the results of random matrix theory, so the connection between the use of random matrix methods for classically chaotic systems is supported by this work.

We begin the application of random matrix theory by determining the average values of the moduli of the matrix elements $\mathbf{J}_{j k}=\langle j|\mathbf{J}| k\rangle$ where $|j\rangle,|k\rangle$ indicate eigenvectors of the unitary baker operator B. We begin with the diagonal elements, which we first express in the position representation of the $N$ quantum states defined in the baker cell. That is, we write

$$
\left|J_{j j}\right|^{2}=|\langle j|J| j\rangle|^{2}=\left(\sum_{\alpha=0}^{N / 2-1}|\langle\alpha \mid j\rangle|^{2}-\sum_{\alpha=N / 2}^{N-1}|\langle\alpha \mid j\rangle|^{2}\right)^{2}
$$

where the kets $|\alpha\rangle$ are basis vectors in position space. Since the eigenkets are normalized, that is, $\sum_{\alpha=0}^{N-1}|\langle\alpha \mid j\rangle|^{2}=1$, we may set $s_{j}=\sum_{\alpha=0}^{N / 2-1}|\langle\alpha \mid j\rangle|^{2}$ to obtain

$$
\left|J_{j j}\right|^{2}=\left(2 s_{j}-1\right)^{2}=4 s_{j}^{2}-4 s_{j}+1
$$


To get the random matrix theory average value, we observe that the joint probability of single eigenstate components in the CUE and COE ensembles are given by $[2,25]$

$$
P(\{\Psi\})=c \delta\left(1-|\Psi|^{2}\right)=c \delta\left(1-\sum_{\alpha=0}^{2 M-1}\left|\Psi_{\alpha}\right|^{2}\right) .
$$

In the COE ensemble the eigenstates can be chosen real which implies that $P(\{\Psi\})$ is a uniform distribution on $N$-dimensional unit sphere, and $M=$ $N / 2$. In CUE ensemble the eigenstates are complex implying that $P(\{\Psi\})$ is uniform on a $2 N$-dimensional unit sphere $\left(\left|\Psi_{j}\right|^{2}=\left(\Re \Psi_{j}\right)^{2}+\left(\Im \Psi_{j}\right)^{2}\right)$, and $M=N$. These averages can be calculated in straightforward ways and one obtains $\langle s\rangle=1 / 2$ for both ensembles. However the averages $\left\langle s^{2}\right\rangle$ differ in the two ensembles. We obtain

$$
\left\langle s^{2}\right\rangle=\frac{\Gamma\left(\frac{M}{2}+2\right)}{\Gamma\left(\frac{M}{2}\right)} \frac{\Gamma(M)}{\Gamma(M+2)}=\frac{M+2}{4(M+1)},
$$

where $M=N / k$ with $k=2$ for the COE and $k=1$ for the CUE. This leads to an evaluation of the averages needed for the diagonal terms, with the result that

$$
\left\langle\left|J_{j j}\right|^{2}\right\rangle=\frac{k}{N+k}
$$

To get the average of the off-diagonal terms, we use (23) which, after averaging, leads to

$$
\left\langle\left|J_{j \neq k}\right|^{2}\right\rangle=\frac{N}{(N-1)(N+k)},
$$

with $k$ given above.

\subsection{The MSD in the CUE ensemble}

Next we calculate the average of $e^{i\left(\varphi_{1}-\varphi_{2}\right) \tau}$ in CUE ensemble. To do this we need an expression for the pair correlation function, $R\left(\varphi_{j}, \varphi_{k}\right)$ of two angles in this ensemble. This is calculated in some detail by Mehta [23], and we use the expression given there.

$$
\left\langle e^{i\left(\varphi_{1}-\varphi_{2}\right) \tau}\right\rangle=\int_{0}^{2 \pi} d \varphi_{1} \int_{0}^{2 \pi} d \varphi_{2} e^{i\left(\varphi_{1}-\varphi_{2}\right) \tau} \frac{R\left(\varphi_{j}, \varphi_{k}\right)}{N(N-1)}
$$




$$
=\int_{0}^{2 \pi} d \varphi_{1} \int_{0}^{2 \pi} d \varphi_{2} e^{i\left(\varphi_{1}-\varphi_{2}\right) \tau} \frac{N}{4 \pi^{2}(N-1)}\left[1-\frac{\sin ^{2} \frac{N\left(\varphi_{j}-\varphi_{k}\right)}{2}}{N^{2} \sin ^{2} \frac{\left(\varphi_{j}-\varphi_{k}\right)}{2}}\right]
$$

At $\tau=0$ the first term is $N /(N-1)$, and it vanishes for $\tau>0$. We calculate the second term by converting the angular integral to one in the complex plane. By changing the variables to $u=\varphi_{1}-\varphi_{2}, v=\varphi_{2}$, and then setting $z=e^{i u}, d z=i z d u$, we obtain, for $\tau>0$,

$$
\begin{aligned}
\left\langle e^{i\left(\varphi_{1}-\varphi_{2}\right) \tau}\right\rangle & =\frac{-1}{2 \pi N(N-1)} \int_{0}^{2 \pi} d u e^{i u \tau} \frac{\sin ^{2} \frac{N u}{2}}{\sin ^{2} \frac{u}{2}} \\
& =\frac{-1}{2 \pi i N(N-1)} \oint d z z^{\tau-N} g(z),
\end{aligned}
$$

where

$$
g(z)=\sum_{p} a_{p} z^{p}=\left(\sum_{k=0}^{N-1} z^{k}\right)^{2}=\sum_{k=0}^{N-1}(k+1) z^{k}+\sum_{k=N}^{2 N-2}(2 N-k-1) z^{k} .
$$

Since $g(z)$ is analytic, then $\left\langle\exp \left[i\left(\varphi_{1}-\varphi_{2}\right) \tau\right]\right\rangle=0$ if $\tau-N \geq 0$. Otherwise, we can write

$$
\frac{1}{2 \pi i} \oint d z z^{\tau-N} g(z)=\frac{1}{p !} g^{(p)}(0)
$$

where $p=N-\tau-1$, and $g^{(p)}$ is the $p$-th derivative of $g$, and obtain

$$
\left\langle e^{i\left(\varphi_{1}-\varphi_{2}\right) \tau}\right\rangle=\frac{-a_{p}}{N(N-1)} .
$$

Thus, including the contribution from the first term at $\tau=0$, we obtain

$$
\left\langle e^{\left[i\left(\varphi_{j}-\varphi_{k}\right) \tau\right]}\right\rangle_{C U E}= \begin{cases}1 & \text { for } \tau=0 \\ \frac{\tau-N}{N(N-1)} & \text { for } 0<\tau<N \\ 0 & \text { for } \tau \geq N .\end{cases}
$$

We now substitute the results, Eqs. (45), (46), and (53) into the expression Eq. (25) the for mean square displacement. This leads to

$$
\left\langle(\Delta r)^{2}\right\rangle=t+t(t-1)\left\langle\left|J_{j j}\right|^{2}\right\rangle+(N-1)\left\langle\left|J_{j \neq k}\right|^{2}\right\rangle \sum_{\tau=1}^{t-1}(t-\tau)\left\langle e^{i \alpha \tau}+e^{-i \alpha \tau}\right\rangle
$$




$$
=t+\frac{1}{N+1} t(t-1)+\frac{2}{(N+1)(N-1)} \sum_{\tau=1}^{M-1}(t-\tau)(\tau-N)
$$

where $M=t$ for $t<N$, and $M=N$ for $t \geq N$. Carrying out the required sums we obtain the final result for the MSD in the CUE ensemble:

$$
\left\langle(\Delta r)^{2}(t)\right\rangle= \begin{cases}t+\frac{t(t-1)}{N+1}\left[\frac{t-2}{3(N-1)}\right] & \text { for } t \leq N, \\ \frac{1}{N+1} t^{2}+\frac{N}{3} & \text { for } t>N .\end{cases}
$$

\subsection{The MSD in the COE ensemble}

The calculation of the MSD in the circular orthogonal ensemble proceeds in very much the same way as in the CUE, the only difference being in the pair

correlation function for the quasi-energies, $\varphi_{j}$. For the COE the two-point correlation function is [see Mehta[23], Eq.(6.2.4), Eq.(10.3.41)]

$$
\begin{aligned}
R_{2}(\vartheta, \varphi) & =\operatorname{det}\left[\begin{array}{cc}
\sigma_{N}(0) & \sigma_{N}(\vartheta-\varphi) \\
\sigma_{N}(\varphi-\vartheta) & \sigma_{N}(0)
\end{array}\right] \\
& =\left[\left(\sigma_{N}(0)\right)^{2}\right]^{(0)}-\left[\left(\sigma_{N}(\vartheta-\varphi)\right)^{2}\right]^{(0)}
\end{aligned}
$$

Here, $\sigma_{N}$ is a quaternion, and det is the quaternion determinant, with

$$
\left[\left(\sigma_{N}(\vartheta-\varphi)\right)^{2}\right]^{(0)}=\left(S_{N}(\vartheta-\varphi)\right)^{2}+D S_{N}(\vartheta-\varphi) J S_{N}(\vartheta-\varphi)
$$

These quantities are given by Mehta as

$$
\begin{aligned}
S_{N}(\vartheta) & =\frac{1}{2 \pi} \frac{\sin \frac{N \vartheta}{2}}{\sin \frac{\vartheta}{2}}=\frac{1}{2 \pi} \sum_{k=0}^{N-1} e^{i p_{k} \vartheta} \\
D S_{N}(\vartheta) & =\frac{1}{2 \pi} \sum_{k=0}^{N-1} i p_{k} e^{i p_{k} \vartheta}=\frac{d}{d \vartheta} S_{N}(\vartheta) \\
J S_{N}(\vartheta) & =-\frac{1}{2 \pi i} \sum_{q} \frac{1}{q} e^{i q \vartheta}
\end{aligned}
$$

where $p_{k}=\frac{1}{2}-\frac{N}{2}+k, \quad k=0,1, \ldots, N-1$, and $q= \pm \frac{1}{2}(N+1), \pm \frac{1}{2}(N+3), \ldots$ Then

$$
\left\langle e^{i(\vartheta-\varphi) \tau}\right\rangle_{C O E}=\frac{1}{N(N-1)}\left[S_{1}(\tau)+S_{2}(\tau)+S_{3}(\tau)\right]
$$


where

$$
\begin{aligned}
& S_{1}(\tau)=\int d \vartheta \int d \varphi e^{i(\vartheta-\varphi) \tau} \frac{N^{2}}{4 \pi^{2}} \\
& S_{2}(\tau)=-\int d \vartheta \int d \varphi e^{i(\vartheta-\varphi) \tau} \frac{1}{4 \pi^{2}} \sum_{k, l=0}^{N-1} e^{i\left(p_{k}+p_{l}\right)(\vartheta-\varphi)}, \\
& S_{3}(\tau)=\int d \vartheta \int d \varphi e^{i(\vartheta-\varphi) \tau} \frac{1}{4 \pi^{2}} \sum_{p, q} \frac{p}{q} e^{i(p+q)(\vartheta-\varphi)}
\end{aligned}
$$

The evaluation of these sums and integrals is relatively straightforward, and leads to

$$
\left\langle e^{[i \alpha \tau]}\right\rangle_{C O E}= \begin{cases}1 & \text { for } \tau=0 \\ \frac{1}{N(N-1)}\left[-N+2 \tau\left[f\left(\frac{N}{2}+\tau\right)-f\left(\frac{N}{2}\right)\right]\right] & \text { for } 0<\tau<N \\ \frac{1}{N(N-1)}\left[-N+2 \tau\left[f\left(\frac{N}{2}+\tau\right)-f\left(\tau-\frac{N}{2}\right)\right]\right] & \text { for } \tau \geq N\end{cases}
$$

Here $f(T)$ is defined by

$$
f(T):=\sum_{k=1}^{T} \frac{1}{2 k-1}=1+\frac{1}{3}+\ldots+\frac{1}{2 T-1} .
$$

This function has at most a logarithmic dependence on its upper limit for large $T$. For the MSD in the COE we then obtain

$$
\left\langle(\Delta r)^{2}(t)\right\rangle= \begin{cases}t+\frac{t(t-1)}{N+2}\left[1+\frac{t-2}{3(N-1)}\right]+\delta_{<} & \text {for } t \leq N, \\ \frac{2}{N+2} t^{2}+\frac{N}{3}-\frac{N}{3(N+2)}+\delta_{>} & \text {for } t>N .\end{cases}
$$

Here $\delta_{<,>}$are small corrections to the explicit formulae that have to be evaluated numerically. Note that the explicit results given here for both ensembles can be combined into the single expression

$$
\left\langle(\Delta r)^{2}(t)\right\rangle= \begin{cases}t+\frac{t(t-1)}{N+k}\left[k-1+\frac{t-2}{3(N-1)}\right]+(k-1) \delta_{<} & \text {for } t \leq N \\ \frac{k}{N+k} t^{2}+\frac{N}{3}-\frac{N(k-1)}{3(N+k)}+(k-1) \delta_{>} & \text {for } t>N .\end{cases}
$$

Those results are shown in Figure 2 for $N=200$. The COE results are two close curves, where the higher is the result given in Eq. (63) for $k=2$, while 
in the lower curve the corrections $\delta_{<,>}$have been neglected. Three asymptotic estimates $t, t^{2} / N, 2 t^{2} / N$ are also plotted. Inset shows the region $t=100$ to $t=300$ where the differences between the two COE results are most pronounced. To our delight, RMT average lends classical diffusion as the short-

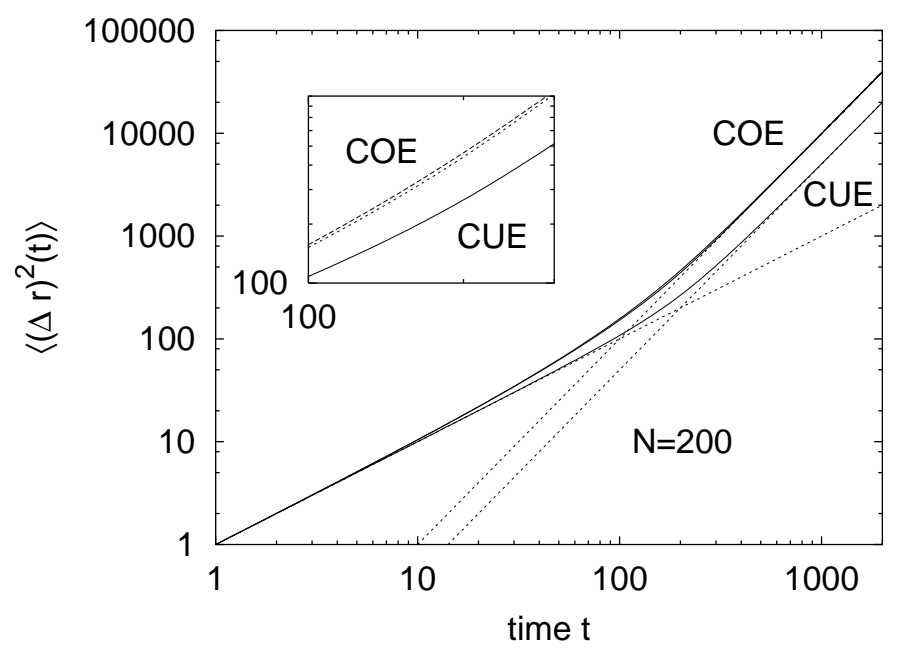

Fig. 2. Log-log plot of the ensemble averages of the mean square displacement using RMT. Both CUE and COE results are plotted. The COE results are the two close curves, where the higher is the result given in Eq. (63) for $k=2$, while in the lower curve the corrections $\delta_{<,>}$have been neglected. Three asymptotic estimates $t, t^{2} / N, 2 t^{2} / N$ are also plotted. Inset shows the region $t=100$ to $t=300$ where the differences between the two COE results are most pronounced.

time prediction in both cases, the CUE average being "more classical". It is worth emphasizing that the classical behavior persists up to the Heisenberg times $\sim h^{-1}=N$ rather than the Ehrenfest time $\sim \ln h^{-1}=\ln N$. On the other hand, for times longer than the Heisenberg time we observe ballistic motion. The ballistic coefficient is proportional to the effective Planck constant therefore it disappears in semi-classical limit.

In other words, fixing the time and performing semi-classical limit $(h \rightarrow 0 \equiv$ $N \rightarrow \infty)$ we obtain $\left\langle(\Delta r)^{2}(t)\right\rangle=t$, which is the classical result relevant for both the classical multi-baker and the $1 \mathrm{D}$ random walk, which is modeled by the classical system. Fixing the Planck constant $(N=$ const $)$ and performing long time limit we observe $\left\langle(\Delta r)^{2}(t)\right\rangle=k t^{2} / N$, which is reflection of the crystal-like structure of the system.

\subsection{Extremal properties of the MSD}

To get another perspective on the above results consider a more general class of quantum multiplexer maps [17] where the local dynamics is given by a different map $B$, e.g a cat map, standard map, or other, replacing the baker map. Then 
the formula (27) is still valid, however the eigenvalues and eigenvectors are those of the new map $B$.

Using formula (27) it is easy to see that the time-dependent mean square displacement for any quantum multiplexer map has to satisfy

(1) $\left\langle(\Delta r)^{2}(0)\right\rangle=0$

(2) $\left\langle(\Delta r)^{2}(1)\right\rangle=1$

(3) $0 \leq\left\langle(\Delta r)^{2}(t)\right\rangle \leq t^{2}$

In fact, those results are true in both the quantum and the classical case. Moreover, it is possible to find local dynamics which realize both of the extremal cases.

To see it, take as the local map a right-left exchange operator, defined classically by

$$
B(n, x, y):= \begin{cases}(n, x+1 / 2, y), & \text { for } 0 \leq x<1 / 2 \\ (n, x-1 / 2, y), & \text { for } 1 / 2 \leq x<1\end{cases}
$$

and quantum mechanically by

$$
\mathbf{B}:=\left[\begin{array}{ll}
0 & 1 \\
1 & 0
\end{array}\right]
$$

(using the context of Section 2). The dynamics is obvious: the particle jumps between two neighboring sites, leading to the mean square displacement having values 0 for even and 1 for odd times.

On the other hand, taking identity as the local dynamics, we induce ballistic motion: particle starting in the state going initially to the right will keep on going to the right, leading to the ballistic transport:

$$
\left\langle(\Delta r)^{2}(t)\right\rangle=t^{2}
$$

Therefore, we see that translational invariance of the coupling operator $T$ allows, in principle, for large asymptotic freedom:

$$
\left\langle(\Delta r)^{2}(t)\right\rangle \propto t^{\alpha}
$$

where $0 \leq \alpha \leq 2^{5}$. An interesting question is, what can be realized in $\overline{5} \mathrm{~A}$ more precise statement is: there exists a constant $\alpha \in[0,2]$ such that 
practice. While no constraints seem to be imposed on the classical level, the structure of the quantum mean square displacement, Eq. (26) suggests that for fixed $\hbar$ only $\alpha=0$ or $\alpha=2$ are viable. One of the questions it raises is what conditions need to be imposed on the internal dynamics so that the semi-classical limit leads to anomalous diffusion $\alpha \neq 1$. We interpret our RMT result as follows: internal fully chaotic dynamics (mixing) of the classical map implies generically diffusion. We are thus led to believe, that anomalous diffusion can arise in semi-classical limit in systems with partially chaotic, partially integrable internal dynamics. Similar observations were made often before in the context of different types of systems and transport in phase space as opposed to the transport in real space, which we discuss here. More precise results require further study.

\section{Comparison of numerical results with random matrix theory pre- dictions}

We have studied numerically a number of cases of quantum multi-bakers as well as some more general systems [17]. Here we present some of those results to show the quality of prediction afforded by the random matrix theory.

Our numerical evaluation of the formula (27) shows that generically, almost every choice of phases defining the quantization (3) leads to results between the COE and CUE results. Figure 3 shows results obtained for the quantum multi-baker $(2)$ with phases $\varphi_{q}=0.73, \varphi_{p}=0.11$. We show the mean square displacement as a function of time for $N=200$ and for $N=2000$ on log-log plot. There is a clear crossover between the diffusive and ballistic behavior on both plots. Figure (3.c) compares the values of $\frac{1}{N} \sum_{j}\left\langle\left|J_{j j}\right|^{2}\right\rangle$ calculated for the same quantum multi-baker for various values of $N$ with the predictions of random matrix theory (45). We see that for $N>50$ the results lie between the analytic values for COE and CUE ensembles.

In cases when the two phases add up to one $\varphi_{q}+\varphi_{p}=1$, we find from our numerical work that the ballistic coefficient $\frac{1}{N} \sum_{j}\left\langle\left|J_{j j}\right|^{2}\right\rangle$ vanishes. Therefore, only the oscillating contribution remains in the expansion (26). We still observe diffusive behavior up to Heisenberg time, although not as clearly as for generic systems. After this transient the particle localizes and the mean square displacement oscillates irregularly around some average value. Figure 4 shows the mean square displacement for quantum multi-baker with phases $\varphi_{q}=\varphi_{p}=1 / 2$ [9] for $N=50$ (top row) and $N=200$ (bottom row). In both cases the left plot shows the short time behavior on the log-log scale. We see the same diffusive behavior as in the generic case. The right plots are

$\overline{\lim }_{t \rightarrow \infty}\left\langle(\Delta r)^{2}(t)\right\rangle / t^{\beta}=\infty$ for $\beta<\alpha$, and $\lim _{t \rightarrow \infty}\left\langle(\Delta r)^{2}(t)\right\rangle / t^{\beta}=0$ for $\alpha<\beta$ 

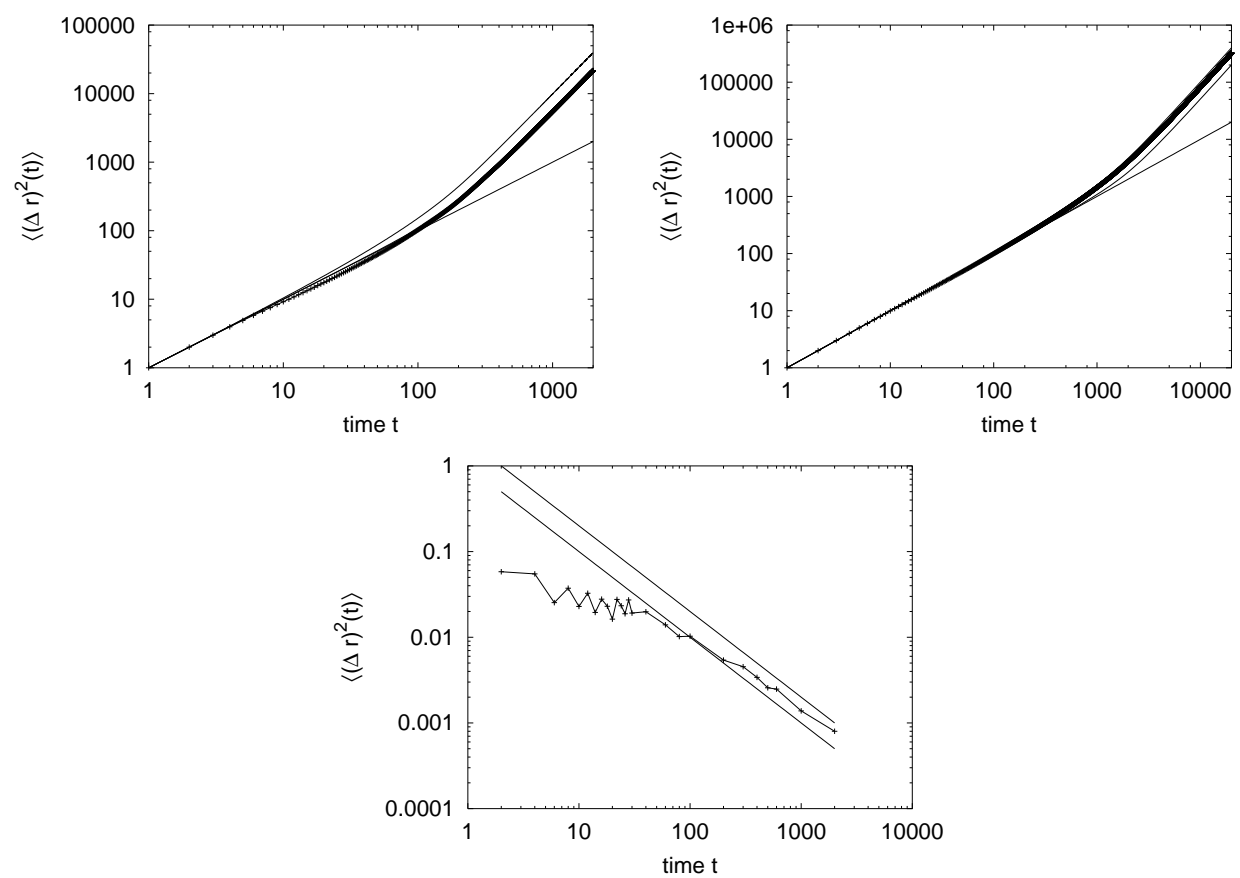

Fig. 3. Log-log plot of the mean square displacement in the quantum multi-baker (2) with phases $\varphi_{q}=0.73, \varphi_{p}=0.11$ as a function of time for $N=200$ (top left) and for $N=2000$ (top right). The crossover between the diffusive and ballistic behavior is clearly visible on both plots at the Heisenberg time $t=N$. The bottom figure shows the values of $\frac{1}{N} \sum_{j}\left\langle\left|J_{j j}\right|^{2}\right\rangle$ calculated for the same quantum multi-baker for various values of $N$ with the predictions of random matrix theory (45). We see that for $N>50$ the results lie between the analytic values for COE and CUE ensembles.

normal scale and show the oscillations encountered on the long scale. All the plots are numerical evaluations of the exact formula (26). We suspect that this non-generic, localized behavior will be seen whenever the phases $\varphi_{q}, \varphi_{p}$ sum to unity, but both the proof and a physical understanding remain to be developed.

\section{Multi-baker maps and quantum random walks}

We conclude this discussion of the quantum multi-baker maps by establishing connections between our work and some other research on quantum random walks. We restrict ourselves to discrete quantum walks on line. Continuous quantum random walks $[26,27,28]$ and discrete quantum walks on graphs $[29,30]$ are less connected to our work.

A popular model of a quantum random walk, known today as the Hadamard walk, was described some ten years ago by S. Godoy and S. Fujita [31]. It was obtained through approximating the evolution of some special wave packets in 

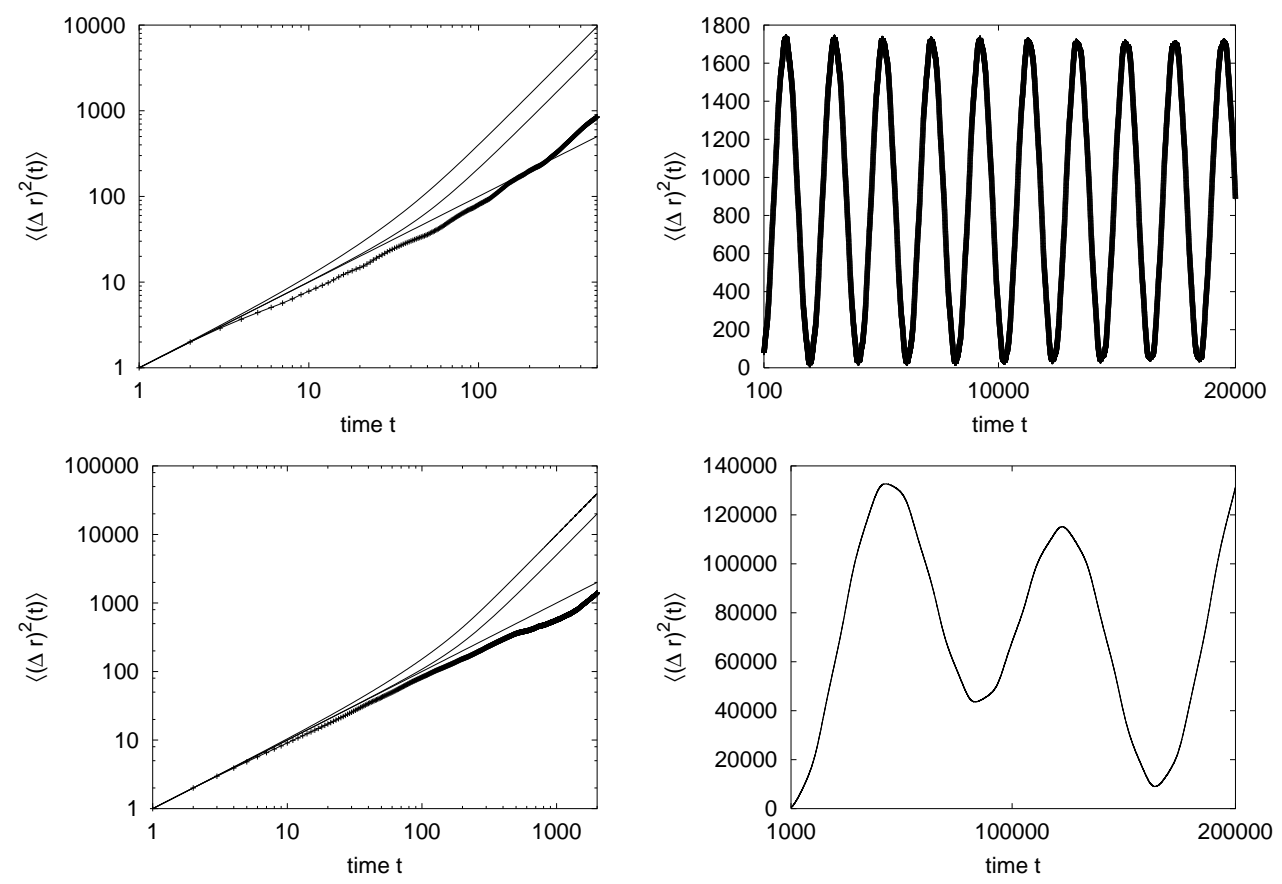

Fig. 4. Mean square displacement for quantum multi-baker with phases $\varphi_{q}=\varphi_{p}=1 / 2$ for $N=50$ (top row) and $N=200$ (bottom row). The left plots show the short time behavior on the log-log scale. We see the same diffusive behavior as in the generic case. The right plots are normal scale and show the oscillations encountered on the long scale. All the plots are numerical evaluations of the exact formula (26).

Krönig-Penney type potential. A similar model was discussed by D. Aharonov, et al. [32], who considered the motion of spin- $\frac{1}{2}$ particles in one dimension, and proposed experimental realization of the walk in the framework of quantum optics. Further, D. Meyer studied quantum cellular automata for their possible applications to quantum computing. In section 5 of [33], Meyer obtained a quantum random walk model closely related to the Hadamard walk, discussed later by A. Ambainis et al. [16].

Looking for an analytically tractable model of quantum transport, we quantized [12] the classical multi-baker map, a deterministic model of classical random walk, obtaining a family of quantum random walk models, the simplest case being the Hadamard walk. We studied both the translationally invariant and random versions of the system.

Since then a number of other papers appeared on the topics, e.g. [34,35,36,37,38,39,40,41,42,43,44,45]. Of particular interest for our work is the work of Brun et al. [46,47,48] who also studied the transition to classical behavior, but instead of considering semi-classical limit they assume interaction with environment implemented in decoherent coins.

Much of the work on discrete quantum random walks can easily be refor- 
mulated as a special case of the multi-baker maps (or multiplexer maps, see ref. [17]) in particular, the map with the largest possible value of Planck's constant, $h=1 / 2$, or equivalently, $N=2$. In order to make this connection we review, very briefly, the structure of quantum random walks as described by much of the literature.

The simplest quantum random walk described in the literature is the Hadamard walk. Here one considers a collection of quantum states, which we will denote as $|n, d\rangle$. The parameter $n$ will take on all integer values within a prescribed range, and here we take the range to be $\mathbf{Z}$, all integers, positive and negative. The parameter $d=r, l$ denotes a direction, to the right or to the left, respectively. Next, one defines an elementary quantum transformation, $\mathbf{H}$, which takes $|n, d\rangle$ into symmetric and anti-symmetric combinations, as

$$
\left(\begin{array}{c}
\left|n, r^{\prime}\right\rangle \\
\left|n, l^{\prime}\right\rangle
\end{array}\right)=\mathbf{H}\left(\begin{array}{c}
|n, r\rangle \\
|n, l\rangle
\end{array}\right)=\frac{1}{\sqrt{ } 2}\left(\begin{array}{cc}
1 & 1 \\
1 & -1
\end{array}\right) \cdot\left(\begin{array}{c}
|n, r\rangle \\
|n, l\rangle
\end{array}\right) .
$$

This step is now coupled to a translation, $\mathbf{T}$ of $\left|n, r^{\prime}\right\rangle$ one unit to the right and, similarly, $\left|n, l^{\prime}\right\rangle$ one unit to the left, as

$$
\begin{aligned}
& \mathbf{T}|n, r\rangle=|n+1, r\rangle \\
& \mathbf{T}|n, l\rangle=|n-1, l\rangle
\end{aligned}
$$

$H$ is called the Hadamard gate in quantum computing literature [49,50], which gives rise to the name Hadamard walk. Finally the two operations are combined to form the operator $\mathbf{T} \circ \mathbf{H}=\mathbf{W}$, which describes one step of the Hadamard walk,

$$
\begin{aligned}
& |n, r, t\rangle=\frac{1}{\sqrt{ } 2}[|n-1, r, t-1\rangle+|n+1, l, t-1\rangle] \\
& |n, l, t\rangle=\frac{1}{\sqrt{ } 2}[|n-1, r, t-1\rangle-|n+1, l, t-1\rangle] .
\end{aligned}
$$

This is identical to Eq. (2) for $N=2$ for the special case that the Balazs-Voros phases [8], $\varphi_{q}=\varphi_{p}=0$, are used. Therefore, the multi-baker map and the Hadamard walk are identical, for the Balazs-Voros phases and $N=2$. The Hadamard equations have been solved in detail for various boundary conditions $[12,16,34,38,45]$. Similarly, the $N=2$ multi-baker map has been solved for a variety of phases and boundary conditions [12]. It should be appreciated that the multi-baker maps represent generalizations of quantum random walks in a number of respects. The inclusion of phases in the transformation equations allows one to treat a variety of maps including uniform, periodic, quasi-periodic, and random systems, something typically not considered in the 
theory of quantum random walks. Thus one can find a range of phenomena in quantum multi-baker ranging from localization to ballistic motion. Such phenomena show up in many condensed matter systems. Further, the use of a variable Planck constant allows for several channels of motion to be taking place at once, and allows us to treat semi-classical as well as strong quantum versions of these maps. This is what makes the multi-baker maps so appealing for studying exact transport properties of condensed quantum systems.

One common feature of different quantum random walks and quantum multibaker maps is the ability to include various models of decoherence processes. In the multi-baker maps one should distinguish between quenched disorder and annealed disorder. In quenched disorder the phases $\varphi_{q}, \varphi_{p}$ vary randomly for cell to cell, but once values are given for each cell, the phases keep those values for all time steps. This allows some memory to be generated by the dynamics and results in localization [12]. For annealed disorder, the phases are chosen randomly for each cell and at each time step. The phases also may be specified up to some additive noise contribution. These processes represent the effects of random external noise and result in some degree of decoherence $[36,46]$. Further, in both quantum random walk models and multi-baker maps one can produce decoherence by replacing the unitary matrix describing the time evolution by a completely positive super-operator. This allows for both dissipation and decoherence. As this topic requires a separate discussion, we will not pursue it here, but leave the discussion of decoherence for further publications.

\section{Discussion of the results and other comments}

This paper described calculations of important properties of the quantum multi-baker map, a simple model for transport in quantum systems. This map is the quantum version of a well known, classically chaotic system. In particular we were able to show, using random matrix theory, that it is possible to obtain an analytic expression for the equilibrium mean square displacement of a particle whose motion is governed by this quantum map. This expression is of particular importance for an understanding of transport in quantum systems because it allows us to carefully examine the particle's average motion both for finite times and for non-zero values of Planck's constant. Thus we can see how the two non-commuting limits, $t \rightarrow \infty$ or $\hbar \rightarrow 0$, interact with each other. While our results are not surprising, in the sense that we already knew qualitatively the limiting forms, we did not have a general expression for the mean square displacement even for simple quantum systems before this work was carried out. Moreover, the results from random matrix theory agreed very well with careful numerical evaluations of the mean square displacement for the multi-baker map. This agreement is also very important because random 
matrix theory does not really apply to any individual system, as far as one knows, but rather to the average behavior of an ensemble of similar systems. Thus in the absence of a rigorous justification of the use of random matrix methods, their predictions must always be checked by comparisons with numerical studies.

It will be interesting to find other signatures of classical behavior in the quantum multi-baker map that are more closely connected to the chaotic behavior of the classical map than the mean square displacement. For example, is there any trace of the classical, hyperbolic dynamical behavior in the quantum version of the map? One prominent feature of the classical multi-baker model is the formation of fractal structures on arbitrarily fine scales as an initial non-equilibrium distribution of points relaxes to a final uniform equilibrium. This fractal structure can, in turn, be related to a positive entropy production typical of the approach to equilibrium of a macroscopic system. The quantum version of this phenomenon is largely unexplored and is of considerable interest to us.

Further work will also be devoted to exploring the consequences of the freedom one has in choosing phases of the unitary operators. This freedom allows us to consider random, quasi-random, and periodic systems, each with its own interesting physical properties. The quenched random system has been explored to some extent [12], and provides a simple model of localization in one-dimensional disordered systems. Here the classical limit is expected to be recovered through a phenomenon where the localization length grows infinitely large as Planck's constant approaches zero, such that almost normal diffusion takes place within each localized region. This picture needs to be verified, and further systems still need to be explored.

We also began to establish connections between this work on quantum multibaker maps and a body of related work on quantum random walk processes. Much of that literature is, in fact, devoted to the simplest multi-baker model, although this connection has not been recognized in the random walk literature. Here we showed that the $N=2$ quantum multi-baker process is identical to the Hadamard walk of random walk theory. Although quantum computing is still far from being realized [49,50], it is possible that the theory of quantum random walks may be of some value for constructing algorithms based on quantum walks that will be faster than current classical random walk algorithms $[27,43]$. Future work will be devoted to expanding the connections between the quantum random walks and multi-baker processes for a variety of model systems. 


\section{Acknowledgments}

The authors would like to thank R. Blume-Kohout and Profs. S. Fishman, P. Gaspard, M. Kuś, S. Tasaki, J. Vollmer, W. Żurek, and K. Życzkowski for helpful comments. DKW is grateful to the Institute for Physical Science and Technology, University of Maryland, where most of this work has been done, for support during his postdoctoral stay; and to the Center for Nonlinear Science, School of Physics, Georgia Institute of Technology for support as a Joseph Ford Fellow. He also thanks the organizing committee of the International Workshop and Seminar on Microscopic Chaos and Transport in ManyParticle Systems, Dresden, 2002 for giving him the opportunity to present this work. JRD acknowledges support from the national Science Foundation under Grant PHY-98-20824.

\section{References}

[1] M. V. Berry, N. L. Balazs, Evolution of semi-classical quantum states in phase space, J. Phys. A: Math. Gen. 12 (1979) 625.

[2] F. Haake, Quantum Signatures of Chaos, 2nd Edition, Springer Verlag, Berlin, New York, 2001.

[3] H. J. Stöckmann, Quantum Chaos. An Introduction, Cambridge University Press, Cambridge, 1999.

[4] R. Alicki, M. Fannes, Quantum dynamical systems, Oxford Univ. Press, Oxford, 2001.

[5] E. Hopf, Ergodentheorie, J. Springer, Berlin, 1937.

[6] J. R. Dorfman, An introduction to chaos in nonequilibrium statistical mechanics, Cambridge University Press, Cambridge, 1999.

[7] P. Gaspard, Chaos, scattering and statistical mechanics, Cambridge University Press, Cambridge, 1998.

[8] N. L. Balazs, A. Voros, The quantized bakers transformation, Ann. Phys. 190 (1989) 1-31.

[9] M. Saraceno, Classical structures in the quantized baker transformation, Ann. Phys. 199 (1990) 37-60.

[10] P. Gaspard, Diffusion, effusion, and chaotic scattering: an exactly solvable Liouvillian dynamics, J. Stat. Phys. 68 (5/6) (1992) 673.

[11] S. Tasaki, P. Gaspard, Fick's law and fractality of nonequilibrium stationary states in a reversible multi-baker map, J. Stat. Phys. 81 (5/6) (1995) 935-987. 
[12] D. K. Wójcik, J. R. Dorfman, Quantum multi-baker maps: Extreme quantum regime, Phys. Rev. E 66 (2002) 036110.

[13] D. K. Wójcik, J. R. Dorfman, Diffusive-ballistic crossover in 1D quantum walks, Phys. Rev. Lett. 90 (2003) 230602.

[14] A. Lakshminarayan, N. L. Balazs, Relaxation and localization in interacting quantum maps, J. Stat. Phys. 77 (1994) 350-373

[15] G. Baym, Lectures on Quantum Mechanics, Benjamin. Publ. Co, Reading, MA, 1969.

[16] A. Ambainis, E. Bach, A. Nayak, A. Vishwanath, J. Watrous, One-dimensional quantum walks, in: Proceedings of the 33rd Annual ACM Symposium on Theory of Computing, Association for Computing Machinery, New York, 2001, pp. 3749 .

[17] D. K. Wójcik, J. R. Dorfman, in preparation (2002).

[18] J. H. Hannay, M. V. Berry, Quantization of linear maps on a torus - Fresnel diffraction by a periodic grating, Physica D 1 (1980) 267.

[19] S. DeBievre, M. D. Esposti, R. Giachetti, Quantization of a class of piecewise affine transformations on the torus, Commun. Math. Phys. 176 (1996) 73-94.

[20] S. De Bievre, M. D. Esposti, Egorov theorems and equidistribution of eigenfunctions for the quantized sawtooth and baker maps, Ann. Inst. Henri Poincare-Phys. Theor. 69 (1998) 1-30.

[21] S. De Biévre, Quantum chaos: a brief visit, in: S. Perez-Esteva, C. VillegasBlas (Eds.), Second Summer School in Analysis and Mathematical Physics: Topics in Analysis: Harmonic, Complex, Nonlinear, and Quantization, Vol. 289 of Contemporary Mathematics, AMS, 2001.

[22] A. Bäcker, Numerical aspects of eigenvalue and eigenfunction computations for chaotic quantum systems, nlin.CD/0204061 (2002).

[23] M. L. Mehta, Random matrices, Academic Press, New York, 1991.

[24] P. W. Oconnor, S. Tomsovic, The unusual nature of the quantum baker transformation, Ann. Phys. 207 (1991) 218-264.

[25] M. Kuś, J. Mostowski, F. Haake, Universality of eigenvector statistics of kicked tops of different symmetries, J. Phys. A 21 (1988) L1073-L1077.

[26] A. M. Childs, E. Farhi, S. Gutmann, An example of the difference between quantum and classical random walks, quant-ph/0103020 (2001).

[27] A. M. Childs, R. Cleve, E. Deotto, E. Farhi, S. Gutmann, D. A. Spielman, Exponential algorithmic speed-up by quantum walk, in: Proceedings of the 35th Annual ACM Symposium on Theory of Computing, Association for Computing Machinery, New York, 2001, pp. 59-68; quant-ph/0209131 (2002). 
[28] A. Ahmadi, R. Belk, C. Tamon, and C. Wendler, Mixing in Continuous Quantum Walks on Graphs, quant-ph/0209106 (2002).

[29] D. Aharonov, A. Ambainis, J. Kempe, U. Vazirani, Quantum walks on graphs, in: Proceedings of the 33rd Annual ACM Symposium on Theory of Computing, Association for Computing Machinery, New York, 2001, pp. 50-59.

[30] P. Leroux, Coassociative grammar, periodic orbits and quantum random walk over $\mathbb{Z}$, quant-ph/0209100 (2002).

[31] S. Godoy, S. Fujita, A quantum random-walk model for tunneling diffusion in a 1d lattice - a quantum correction to Fick's law, J. Chem. Phys. 97 (1992) $5148-5154$.

[32] Y. Aharonov, L. Davidovich, N. Zagury, Quantum random-walks, Phys Rev A 48 (2) (1993) 1687-1690.

[33] D. A. Meyer, From quantum cellular automata to quantum lattice gases, J. Stat. Phys. 85 (1996) 551-574.

[34] E. Bach, S. Coppersmith, M. P. Goldschen, R. Joynt, J. Watrous, Onedimensional quantum walks with absorbing boundaries, quant-ph/0207008 (2002).

[35] W. Dür, R. Raussendorfer, V. M. Kendon, H.-J. Briegel, Quantum walks in optical lattices, Phys. Rev. A 66 (2002) 052319.

[36] V. Kendon, B. Tregenna, Decoherence is useful in quantum walks, Phys. Rev. A 67 (2003) 042315 .

[37] V. Kendon, B. Tregenna, Decoherence in a quantum walk on the line, quant-ph/0210047 (2002).

[38] N. Konno, Quantum random walks in one dimension, quant-ph/0206053 (2002).

[39] N. Konno, A new type of limit theorems for the one-dimensional quantum random walk, quant-ph/0206103 (2002).

[40] N. Konno, T. Namiki, T. Soshi, A. Sudbury, Absorption problems for quantum walks in one dimension, J. Phys. A 36 (2003) 241-253 .

[41] N. Konno, Limit theorems and absorption problems for quantum random walks in one dimension, Quantum Information and Computation 2 SI (2002) 578-595.

[42] T. D. Mackay, S. D. Bartlett, L. T. Stephenson, B. C. Sanders, Quantum walks in higher dimensions, J. Phys. A-Math. Gen. 35 (2002) 2745-2753.

[43] N. Shenvi, J. Kempe, K. B. Whaley, Quantum random walk search algorithm, Phys. Rev. A 67 (2003) 052307.

[44] B. C. Travaglione, G. J. Milburn, Implementing the quantum random walk, Phys. Rev. A 65 (2002) 032310.

[45] T. Yamasaki, H. Kobayashi, H. Imai, Analysis of absorbing times of quantum walks, quant-ph/0205045 (2002). 
[46] T. A. Brun, H. A. Carteret, A. Ambainis, The quantum to classical transition for random walks, quant-ph/0208195 (2002).

[47] T. A. Brun, H. A. Carteret, A. Ambainis, Quantum walks driven by many coins, Phys. Rev. A 67 (2003) 052317.

[48] T. A. Brun, H. A. Carteret, A. Ambainis, Quantum random walks with decoherent coins, Phys. Rev. A 67 (2003) 032304.

[49] J. Preskill, Lecture notes for the course Quantum Computation, www.theory.caltech.edu/people/preskill/ph219 (1999).

[50] M. A. Nielsen, I. L. Chuang, Quantum computation and quantum information, Cambridge University Press, Cambridge, 2000. 